\title{
Fundamentos tecnológicos de las nuevas normas alemanas del hormigón
}

\section{R E S U M E N}

Este trabajo hace un breve comentario de la nueva edición de las prescripciones sobre el hormigón desde un punto de vista tecnológico, de las modificaciones con relación a los proyectos (hojas amarillas) y las razones que han conducido a las nuevas prescripciones. Por la extensión del proyecto no ha sido posible abordar todos los detalles. La nueva DIN 1045 contiene prácticamente todas las prescripciones tecnológicas relativas al hormigón de textura cerrada. Para el hormigón ligero armado permanecerán provisionalmente directrices particulares, $y$ para el hormigón pretensado, la norma DIN 4227 tendrá en consideración nuevas exigencias.

En la revisión de todas las prescripciones sobre hormigón, se ha tratado de tener en cuenta lo más posible los conocimientos actuales y el estado del desarrollo práctico. Se han hecho importantes modificaciones en cuanto a los nuevos tipos, introduciendo los procesos de fabricación de los hormigones B I y B II y la garantía de sus propiedades, así como la adopción de exigencias relativas a los hormigones con propiedades particulares. También se han ampliado las condiciones exigidas para la protección de las armaduras contra la corrosión y la realización de ensayos. Se exige un control de calidad en lo que se refiere a los componentes del hormigón, el hormigón B II, productos prefabricados y hormigón preamasado; se trata de un autocontrol y de un control exterior.

\section{GENERALIDADES}

Las actuales determinaciones y ensayos sobre el hormigón proceden en su mayor parte de los años 40. Claro está que siempre se intentó, con ayuda de directrices, decretos oficiales y ediciones de normas, ajustar detalles en los aspectos que habían cambiado con el tiempo. Pero, sin embargo, la concepción básica y las relaciones esenciales han permanecido prácticamente invariables desde los años 40. Como quiera que, como ha ocurrido en los materiales de construcción, la tecnología del hormigón y su análisis habían progresado en su desarrollo y se disponía de nuevos conocimientos que ya habían sido utilizados, fue necesario hacer una refundición de las determinaciones sobre este material. Véase entre otros $(1,2)$. 
Por todo esto, hace unos 12 años, se inició la revisión de las determinaciones sobre el hormigón y las normas relacionadas con ellas. En el ámbito de la DIN 1045, la comisión alemana del hormigón armado puso en funcionamiento 11 grupos de trabajo, que elaboraron proyectos para sectores parciales. Estos proyectos fueron tratados en muchos aspectos en una comisión coordinadora y fueron la base del proyecto total que confeccionó la Comisión Alemana del Hormigón Armado, asesorada en los ambientes técnicos del ramo. En estos círculos técnicos fueron deliberadas también las objeciones al proyecto amarillo de la DIN 1045 publicada en 1968. La nueva versión de la DIN 1045 decidida por la Comisión Alemana del Hormigón Armado se publica en el calendario del hormigón 1971, parte 2, y aparece también en 1971 como impreso blanco.

Paralelamente a esto, en comisiones especiales de trabajo se elaboraron los nuevos reglamentos relacionados con DIN 1045; entre otros, por ejemplo, la norma de cemento DIN 1146, la norma de áridos DIN 4226, la norma de aguas, suelos y gases que atacan al hormigón DIN 4030 y la norma de ensayo del hormigón DIN 1048, porque existían influencias recíprocas en los fundamentos de todas estas normas. Las nuevas versiones de DIN 1146 y de DIN 4030 se publicaron ya, y las nuevas ediciones de la DIN 4226 y de la DIN 1048 se terminaron de deliberar en las comisiones respectivas y serán publicadas en breve.

Para la nueva elaboración de estas normas se establecieron, en primer lugar, los siguientes extremos:

a) La nueva versión de DIN 1045 debía contener todas las determinaciones sobre tecnología del hormigón necesarias para la construcción con hormigón y hormigón armado. Por lo tanto, había que incluir no sólo los más recientes desarrollos, sino también las determinaciones competentes de otros reglamentos (como por ejemplo DIN 1046, DIN 1047, DIN 4030, DIN 4225, directrices para el hormigón preamasado, decreto sobre protección contra la corrosión).

b) La nueva versión de DIN 1045 debía servir únicamente para hormigón con textura cerrada, pero, sin embargo, no para hormigón con grandes huecos ni para hormigones gaseados o espumados. Por consiguiente, el hormigón ligero armado, esencialmente, también pertenece a la DIN 1045; las directrices para hormigón ligero armado debieran mantenerse, sin embargo, en forma de tentativa hasta que se disponga de más experiencias sobre el mismo. Para hormigón con gran porosidad se revisa la DIN 4232.

c) Los fundamentos tecnológicos del hormigón para hormigón pretensado no deberían incluirse en DIN 1045, sino en DIN 4227.

d) La nueva versión de la norma de áridos DIN 4226 debiera de contener todos los áridos para el hormigón (antes: áridos del hormigón).

e) La nueva DIN 4030 debería tratar únicamente del dictamen de aguas, suelos y gases que atacan al hormigón; las medidas constructivas deberían incluirse en DIN 1045.

if) En DIN 1048 debían describirse únicamente los métodos de ensayo, es decir, la realización de pruebas. Las determinaciones sobre tipo y número de pruebas necesarias en un caso concreto debían incluirse en DIN 1045 o bien en DIN 1084.

A continuación se van a detallar las modificaciones tecnológicas más esenciales de las nuevas disposiciones referentes al hormigón. Frente al proyecto amarillo de la DIN 1045 [véase también (3)], se hicieron todavía una serie de modificaciones. 


\section{TIPOS DE RESISTENCIA DEL HORMIGON}

Para el valor característico de la "calidad del hormigón" que se caracteriza por la resistencia a la compresión del hormigón de la DIN 1045 actual, se incluyó en la nueva versión de DIN 1045 el concepto "tipo de resistencia del hormigón". Como quiera que el tipo de resistencia del hormigón tiene un significado algo diferente que la calidad actual del hormigón (véase apartado 2.1), para diferenciar de la nomenclatura actual, en la designación del tipo resistente se sustituyó la letra B que se halla delante del número por $\mathrm{Bn}$.

\subsection{Definición}

La DIN 1045 actual entendía por calidad del hormigón la resistencia media a la compresión a los 28 días de tres cubos de $20 \mathrm{~cm}$ de lado almacenados 7 días en húmedo y 21 días al aire ambiente entre $15^{\circ}$ y $22^{\circ} \mathrm{C}$. Podían resultar valores aislados hasta un $15 \%$ por debajo de la resistencia nominal.

Con los actuales conocimientos de la estadística ya no se considera suficiente una referencia a valores medios al enjuiciar la calidad del hormigón. Como quiera que la rotura eventual de un elemento de construcción siempre tiene lugar en el punto más débil de la zona de cargas elevadas, el dimensionado debiera basarse en una resistencia a la compresión que se consiga o se sobrepase en casi todos los puntos del elemento de construcción. Por este motivo, en la nueva versión de DIN 1045, la resistencia nominal y el dimensionado se coordinaron a una resistencia mínima que, desde el punto de vista estadístico, se basa en la fractila inferior de $5 \%$ del conjunto fundamental. De acuerdo con la valoración estadística de numerosos resultados de ensayo [véase también $(4,5,6)$ ], el valor mínímo (fractila de $5 \%$ ) del valor medio de 3 probetas se halla $50 \mathrm{kp} / \mathrm{cm}^{2}$ por encima de la fractila inferior de $5 \%$ en todos los valores aislados del conjunto fundamental. Esto significa que, con un mismo hormigón, la resistencia nominal de los tipos resistentes de la nueva versión es, en general, $50 \mathrm{kp} / \mathrm{cm}^{2}$ menor que la resistencia nominal de la calidad del hormigón de la versión antigua, o sea que, por ejemplo, un hormigón de la nueva clase de resistencia Bn 250 corresponde aproximadamente, a un hormigón de la calidad B 300 antigua. Mediante este proceder ya no disminuirá más la seguridad de la construcción con dispersiones mayores, es decir, con un hormigón irregular, sino que aumentarán la medida de predicción que el fabricante de hormigón ha de cumplir frente a la resistencia mínima (base del dimensionado) a fin de llegar a los requisitos exigidos.

\subsection{Subdivisión}

La DIN 1045 contenía hasta ahora las calidades de hormigón B 120, B 160, B 225 y B 300 , y la DIN 1047, las calidades B 50 y B 80. Esta subdivisión, que en parte sigue una serie geométrica, es demasiado limitada y no satisface las exigencias actuales. A esto hay que añadir que hoy día las calidades B 120 y B 160 ya no tienen más que una importancia en general muy pequeña para las construcciones de hormigón armado. Con la gran resistencia de los cementos actuales y los contenidos mínimos de cemento a mantener para crear una protección anticorrosiva, un hormigón de composición corriente y fabricado con arreglo a la técnica con textura cerrada alcanza siempre una resistencia a la compresión ge- 
neralmente mayor. Asimismo, la protección anticorrosiva de la armadura podría no ser siempre suficiente en hormigón de las calidades B 120 y B 160. Además, en la nueva versión de DIN 1045 había que tener también en cuenta las calidades del hormigón de más de B 300, que se utilizan desde hace tiempo y se han establecido ya en las disposiciones actuales para piezas prefabricadas de hormigón armado (DIN 4225), para hormigón pretensado (DIN 4227): y para puentes macizos (DIN 1075).

Las reflexiones sobre la subdivisión más conveniente de los tipos resistentes de hormigón condujeron a las dos propuestas siguientes:

a) Bn 50, Bn 100, Bn 200 , Bn 300 , Bn 400 , Bn 500, Bn 600 ;

b) Bn 50, Bn 100, Bn 150, Bn 250, Bn 350, Bn 450, Bn 550 .

Ambas propuestas contienen los tipos Bn 50 y Bn 100 y evitan una subdivisión demasiado acusada. En la propuesta a) impera la serie de centenas enteras. Pero se consideró como inconveniente que para el hormigón armado en obras sencillas sólo se dispone del tipo resistente $\mathrm{Bn} 200$, que según la interpretación antigua corresponde a un $\mathrm{Bn} 250$, y que el B 300 antiguo no existe en esta propuesta, ya que el Bn 300 correspondería a un B 350 antiguo. Pero se creyó que del B 300 antiguo, es decir del nuevo Bn 250, no se podría prescindir, ya que en zonas de árido más rico en arena o algo menos consistente se le puede fabricar todavía sin medidas adicionales caras. Por este motivo las correspondientes comisiones técnicas se decidieron, después de muchas reflexiones, por la propuesta $b$ ). Sus ventajas son, en primer lugar, que contiene dos tipos de resistencia (Bn 150 y Bn 250) para hormigón armado en obras sencillas y, en segundo lugar, que con el Bn 250 se conserva la antigua B 300 y con el Bn 350 la vieja calidad de hormigón para carreteras B 400 .

\subsection{Disposiciones de la nueva versión de DIN 1045}

El hormigón se divide en tipos resistentes Bn 50 a Bn 550 de acuerdo con su resistencia a la compresión determinada a la edad de 28 días en cubos de $20 \mathrm{~cm}$ de arista. Las demás disposiciones sobre los futuros tipos resistentes se deducen de la tabla 1. A causa de la protección anticorrosiva de la armadura, los tipos resistentes Bn 50 y Bn 100 están previstos únicamente para hormigón en masa; el hormigón armado no empieza hasta el tipo Bn 150, que corresponde aproximadamente al B 200 de la clasificación antigua. Como muestra la comparación de las columnas 2 y 3 ó bien 3 y 4 de la citada tabla 1 , el valor mínimo de la resistencia a compresión de cada cubo es igual a la resistencia nominal del tipo resistente y el valor mínimo de la resistencia media a compresión de cada serie de cubos es de $30 \mathrm{kp} / \mathrm{cm}^{2}$ en el $\mathrm{Bn} 50$, y de $50 \mathrm{kp} / \mathrm{cm}^{2}$ en los demás tipos resistentes, mayor que la resistencia nominal. En la prueba de la calidad hay que tomar los 3 cubos de una serie sucesiva de tres cargas de hormigonera diferentes repartidas durante el período de hormigonado; en el hormigón preamasado, siempre que sea posible, de distintos suministros de la misma clase de hormigón (tipo resistente, composición, fabricación).

Los requisitos de resistencia a la compresión para la prueba de la calidad (DIN 1045, apartado 7.4.3.5.2) se consideran cumplidos cuando la resistencia a la compresión de cada cubo alcanza, como mínimo, los valores de la columna 3 (resistencia nominal) y cuando la resistencia media a la compresión de cada serie de cubos alcanza, como mínimo, los valores de la columna 4 de la tabla 1 . Si se ensayan numerosos cubos de hormigón de 
igual composición y fabricación, en cada caso, uno de cada nueve cubos consecutivos puede no llegar a la resistencia nominal (tabla 1, columna 3).

T A B L A 1

Tipos de resistencia del hormigón según DIN 1045

\begin{tabular}{|c|c|c|c|c|c|c|}
\hline & 1 & 2 & 3 & 4 & 5 & 6 \\
\hline Línea & $\begin{array}{c}\text { Grupo de } \\
\text { hormigones }\end{array}$ & $\begin{array}{c}\text { Tipo de } \\
\text { resistencia }\end{array}$ & $\begin{array}{l}\text { Resistencia nominal } * \text { ) } \\
\beta_{w N} \\
\text { (valor mínimo de la re- } \\
\text { sistencia a la compre- } \\
\text { sión } \beta_{\mathrm{w} 28} \text { de cada cubo) }\end{array}$ & $\begin{array}{l}\text { Resistencia de series } \\
\beta_{w s} \\
\text { (valor mínimo de la re- } \\
\text { sistencia media a la } \\
\text { compresión } \beta_{w M} \text { de cada } \\
\text { serie de cubos) }\end{array}$ & $\begin{array}{l}\text { Fabricación } \\
\text { según }\end{array}$ & Aplicaciones \\
\hline- & - & - & $\mathbf{k p} / \mathbf{c m}^{2}$ & $\mathbf{k p} / \mathbf{c m}^{2}$ & - & - \\
\hline 1 & \multirow{4}{*}{$\begin{array}{l}\text { Hormigón } \\
\text { B I }\end{array}$} & $\mathrm{Bn} \quad 50$ & 50 & 80 & \multirow{4}{*}{$\begin{array}{l}\text { Apartado } \\
6.5 .5 \text { de la } \\
\text { DIN } 1045\end{array}$} & \multirow{2}{*}{$\begin{array}{l}\text { Sólo para } \\
\text { hormigón } \\
\text { sin armar }\end{array}$} \\
\hline 2 & & $\mathrm{Bn} 100$ & 100 & 150 & & \\
\hline 3 & & $\mathbb{B n} 150$ & 150 & 200 & & \multirow{5}{*}{$\begin{array}{l}\text { Para hor- } \\
\text { migón sin } \\
\text { armar y } \\
\text { armado }\end{array}$} \\
\hline 4 & & Bn 250 & 250 & 300 & & \\
\hline 5 & \multirow{3}{*}{$\begin{array}{l}\text { Hormigón } \\
\text { B II }\end{array}$} & Bn. 350 & 350 & 400 & \multirow{3}{*}{$\begin{array}{l}\text { Apartado } \\
6.5 .6 \text { de la } \\
\text { DIN } 1045\end{array}$} & \\
\hline 6 & & Bn 450 & 450 & 500 & & \\
\hline 7 & & Bn 550 & 550 & 600 & & \\
\hline
\end{tabular}

*) La resistencia nominal se basa en la fractila de $5 \%$ de la totalidad.

La DIN 1084 (actualmente en proyecto) que, en lugar de los apéndices a DIN 1045 previstos al principio, trata del control de los tipos de hormigón B II de prefabricados de hormigón y de hormigón premezclado, recomienda la valoración estadística de los resultados del control. Con valoración estadística, en obras que consumen por lo menos 1.000 $\mathrm{m}^{3}$ hormigón $\mathrm{B}$ II del mismo tipo en fábricas de prefabricados y en fábricas de hormigón preamasado, las exigencias pueden diferir de los requisitos de resistencia a la compresión de la DIN 1045, apartado 7.4.3.5.2. Como la resistencia nominal de los tipos resistentes se basa en la fractila de $5 \%$ de la totalidad fundamental, basta demostrar que la fractila de $5 \%$ de los resultados de la resistencia a compresión de hormigón de composición y fabricación aproximadamente iguales (tabla 1, columna 3) no queda por debajo de la resistencia nominal (tabla 1, columna 3). Según DIN 1084 (actualmente en proyecto), queda hecha la demostración, si los resultados de 35 probetas fabricadas consecutivamente cumplen la siguiente condición [véase también (6)]:

$$
\beta_{5 \%}=\beta-1,64 \cdot s \geqslant \beta_{w N} .
$$

En esta ecuación, significan:

$\beta_{5 \%}=$ fractila de $5 \%$ de los resultados a base de las líneas características de hipótesis según (6).

$\beta=$ valor medio de los ensayos realizados al azar para $\mathrm{n}=35$.

$\mathrm{s} \quad=$ desviación standard de los ensayos realizados al azar para $\mathrm{n}=35$.

$\beta_{\mathrm{wN}}=$ resistencia nominal del tipo de hormigón según tabla 1 , columna 3 . 
Para ensayar la resistencia del hormigón a la compresión, en lugar de los cubos de $20 \mathrm{~cm}$ de arista pueden utilizarse también otras probetas. Para una fabricación y conservación homogéneas, entre la resistencia a la compresión del cubo de $20 \mathrm{~cm}\left(\beta_{\mathrm{w}}\right)$ y la resistencia a la compresión del cilindro de $15 \mathrm{~cm}$ de diámetro y $30 \mathrm{~cm}$ de altura $\left(\beta_{\mathrm{c}}\right)$ pueden admitirse las siguientes relaciones:

- para los tipos resistentes Bn 150 y menores:

$$
\beta_{\mathrm{w}}=1,25 \cdot \beta_{\mathrm{c}}
$$

- para los tipos resistentes Bn 250 y mayores:

$$
\beta_{\mathrm{w}}=1,18 \cdot \beta_{\mathrm{c}} \text {. }
$$

Si se emplean cubos y cilindros de otro tamaño, se demostrará la relación de la resistencia a la compresión con respecto al cubo de $20 \mathrm{~cm}$, en hormigones de cualquier composición, resistencia y edad, con 6 probetas por lo menos de cada tipo.

\section{GARANTIA DEL TIPO RESISTENTE DEL HORMIGON (PROCEDIMIENTO DE FABRICACION DEL HORMIGON)}

En la refundición de la DIN 1045 hubo temores con respecto a como podía garantizarse el tipo de resistencia requerida del hormigón.

La DIN 1045 antigua contiene determinados requisitos para la adición y composición granulométrica del árido y para el contenido mínimo de cemento, que se había fijado por motivos de protección anticorrosiva. Para las calidades de hormigón B 225 y B 300 se exigieron pruebas de idoneidad, y para las calidades de hormigón B 160 pruebas de calidad.

T A B L A 2

\begin{tabular}{|c|c|c|}
\hline Hormigón B I & \multirow{3}{*}{$\begin{array}{l}\text { Poco volumen de ensayos para } \\
\text { calidad o control. } \\
\text { Exigencias moderadas a la obra } \\
\text { y a la empresa. }\end{array}$} & \\
\hline \multirow[t]{2}{*}{$\begin{array}{l}\text { Tipos de resistencia } \mathrm{Bn} 50 \\
\text { hasta } \mathrm{Bn} 250 \text { inclusive. }\end{array}$} & & $\begin{array}{l}\text { Composición del hormigón se- } \\
\text { gún contenidos mínimos de } \\
\text { cemento. }\end{array}$ \\
\hline & & $\begin{array}{l}\text { Composición del hormigón se- } \\
\text { gún prueba de idoneidad con } \\
\text { medida de predicción deter- } \\
\text { minada. } \\
\text { (Para prefabricados de hor- } \\
\text { migón no se ha establecido la } \\
\text { medida de predicción). }\end{array}$ \\
\hline Hormigón B II & & \\
\hline $\begin{array}{l}\text { Tipos de resistencia } \mathrm{Bn} 350 \\
\text { y mayores. }\end{array}$ & $\begin{array}{l}\text { Control amplio (propio y ajeno), } \\
\text { exigencias mayores a obra y } \\
\text { empresa. }\end{array}$ & $\begin{array}{l}\text { Composición del hormigón (re- } \\
\text { lación agua/cemento) según } \\
\text { prueba de idoneidad. }\end{array}$ \\
\hline
\end{tabular}

Condiciones para la fabricación del hormigón según DIN 1045 
Con arreglo a los conocimientos actuales, para la fabricación del hormigón se ofrecen fundamentalmente dos posibilidades. En obras sencillas con poco control la resistencia necesaria se alcanza del modo más seguro mediante una receta de hormigón establecida previamente del lado de la seguridad. Fábricas de hormigón y obras, cuya instalación y personal satisfacen exigencias mayores, pueden determinar su composición del hormigón en virtud de una prueba de idoneidad. La utilización posterior de esta composición exige un minucioso control de las propiedades del hormigón. Claro está que entre estas dos posibilidades extremas puede haber soluciones intermedias. Las disposiciones de la nueva versión de DIN 1045 para las condiciones de fabricación del hormigón se deducen de la tabla 2. Se distinguen los grupos de hormigón B I y B II. Con respecto al proyecto amarillo de DIN 1045 se establecieron algunas modificaciones.

\subsection{Hormigón B I}

Al grupo de hormigones B I pertenecen los hormigones de los tipos resistentes $\mathrm{Bn} 50$ a Bn 250 inclusive. Existen dos posibilidades para la fabricación de estos hormigones. De un lado, los hormigones pueden fabricarse con los contenidos mínimos de cemento de la tabla 3. Estos contenidos mínimos de cemento son altos, con los que la resistencia exigida al hormigón se alcanza con seguridad y, en hormigón armado, queda asegurada también la protección de la armadura. Los contenidos mínimos de cemento de la tabla 3 , con cemento 250 (DIN 1164) hay que aumentarlos un $15 \%$ y con un tamaño máximo del árido de $16 \mathrm{~mm} 10 \%$ y de $8 \mathrm{~mm} 20 \%$; con cemento 450 (DIN 1164) deben disminuirse como máximo $10 \%$ y con un tamaño máximo del árido de $63 \mathrm{~mm}$, como máximo $10 \%$. En hormigón armado, sin embargo, nunca debe quedar por debajo de $240 \mathrm{~kg} / \mathrm{m}^{3}$. Los contenidos mínimos de cemento se calcularon utilizando una resistencia del cemento de la gama inferior y valores de orientación normales del contenido de agua sobre la ley de la relación agua/cemento; estos ensayos fueron comprobados múltiples veces. Como quiera que los contenidos mínimos de cemento se hallan en el lado de la seguridad, puede ocurrir que la resistencia requerida se sobrepase considerablemente con este procedimiento de fabricación.

T A B L A 3

Contenido mínimo de cemento para hormigón B I con cemento 350 (DIN 1164) y tamaño maximo del árido $32 \mathrm{~mm}$

\begin{tabular}{|c|c|c|c|c|}
\hline \multirow{2}{*}{$\begin{array}{l}\text { Tipos resistentes } \\
\text { de los hormigones }\end{array}$} & \multirow{2}{*}{$\begin{array}{l}\text { Zona de curvas } \\
\text { de tamizado }\end{array}$} & \multicolumn{3}{|c|}{ Contenido de cemento en la mezcla $\left(\mathrm{kg} / \mathrm{m}^{3}\right)$} \\
\hline & & Consistencia $\mathbf{K} 1 *$ ) & Consistencia $\mathbf{K} 2$ & Consistencia $\mathbf{K} 3$ \\
\hline \multirow{2}{*}{ Bn $50 *$ ) } & $\mathrm{A} / \mathrm{B}_{32}$ & 140 & 160 & 二 \\
\hline & $\mathrm{B} / \mathbf{C}_{32}$ & 160 & 180 & - \\
\hline \multirow{2}{*}{ Bn $100 *)$} & $\mathbf{A} / \mathbf{B}_{32}$ & 190 & 210 & 230 \\
\hline & $\mathrm{B} / \mathbf{C}_{32}$ & 210 & 230 & 260 \\
\hline \multirow{2}{*}{ Bn 150} & $\mathbf{A} / \mathbf{B}_{32}$ & 240 & 270 & 300 \\
\hline & $\mathrm{B} / \mathrm{C}_{32}$ & 270 & 300 & 330 \\
\hline \multirow{2}{*}{ Bn 250} & $\mathbf{A} / \mathbf{B}_{32}$ & 280 & 310 & 340 \\
\hline & $\mathrm{B} / \mathrm{C}_{32}$ & 310 & 340 & 380 \\
\hline
\end{tabular}

*) Sólo para hormigón en masa. 
Con la segunda posibilidad de fabricación del hormigón B I, la composición precisa para lograr la resistencia se determinará a base del ensayo de idoneidad. En hormigón armado y por motivos de protección anticorrosiva se observarán, además, los valores límite del apartado 5. Esta prueba de idoneidad es siempre necesaria con hormigón B I si la composición del hormigón no corresponde a la tabla 3 o si en su fabricación se emplean conglomerantes mixtos, aditivos, o sustancias accesorias no minerales o que hay que incluir en el contenido de aglomerantes. Los requisitos de resistencia a la compresión de la prueba de idoneidad se consideran cumplidos cuando la resistencia media a la compresión de 3 cubos de la mezcla de hormigón, para la ejecución de la obra o para la fabricación continua del hormigón, excede de la resistencia de serie (tabla 1, columna 4), por lo menos $30 \mathrm{kp} / \mathrm{cm}^{2}$ para el hormigón $\mathrm{Bn} 50 \mathrm{y}$, por lo menos, $50 \mathrm{kp} / \mathrm{cm}^{2}$ desde el $\mathrm{Bn} 100$ hasta el Bn 250 ambos inclusive. La consistencia se encontrará en el límite superior de la gama de consistencia elegida (límite superior de las dimensiones de una torta de hormigón o límite inferior de la medida de concentración).

En ambos procedimientos de fabricación del hormigón B I sólo se requiere un volumen moderado para el ensayo de la idoneidad o control (apartado 8). El volumen es, en parte, menor que en la DIN 1045 antigua. En obras y empresas tampoco se exigen requisitos más que moderados (DIN 1045, apartado 5.2.1). Para las exigencias a fábricas de prefabricados de hormigón ver el apartado 3.3 y para fábricas de hormigón preamasado el apartado 3.4. En obras de hormigón B I, debe trabajarse únicamente con hormigón de los tipos resistentes Bn 50 hasta $\mathrm{Bn} 250$ inclusive.

\subsection{Hormigón B II}

Al grupo de hormigones, hormigón B II, pertenecen todos los hormigones de los tipos resistentes Bn 350 hasta Bn 550. La composición necesaria para obtener la resistencia adecuada y la relación agua/cemento, hay que determinarlas en estos hormigones a base de su prueba de idoneidad. Para hormigón B II no se ha fijado la predicción a un valor determinado, y debe ser tan amplia que los requisitos de resistencia de la prueba de la idoneidad (apartado 2.3) tienen que cumplirse con seguridad. La medida de predicción puede, por consiguiente, hacerse tanto más pequeña cuanto más uniformemente se fabrica el hormigón. Para el contenido de cemento y la relación agua/cemento en hormigón armado hay que tener también en cuenta valores límite, que no deben sobrepasarse para asegurar la protección anticorrosiva (apartado 5).

La mayor libertad en la elección de la composición del hormigón y el mayor aprovechamiento del mismo, que como consecuencia de ello es posible, presuponen un control minucioso de las propiedades del hormigón y mayores exigencias a la obra y a la empresa. Por lo tanto, en la nueva versión de DIN 1045 para hormigón B II no sólo se requiere un mayor volumen de ensayo, sino también un control minucioso de la calidad, a base de controles propios y al exterior. Otros detalles sobre el control de la calidad del hormigón B II puede verse en DIN 1084, hoja 1 (actualmente en proyecto).

En el hormigón B II se exigen también mayores requisitos a la obra y la empresa (DIN 1045, apartado 5.2.2). Sobre todo, es preciso mencionar que la empresa debe disponer de un centro permanente de ensayos de hormigón, situado de modo que sea posible una estrecha colaboración con las obras a realizar. Este centro de pruebas, que ha de estar equipado con todos los aparatos necesarios para la prueba de idoneidad y de la calidad de hormigón B II, tiene, especialmente, las siguientes misiones: 
a) realización de la prueba de idoneidad del hormigón;

b) realización de la prueba de calidad y de idoneidad, siempre que no hayan sido efectuadas por personal de la obra;

c) inspección del equipo de aparatos y de la fabricación del hormigón de obra;

d) dictamen y valoración de los resultados de todas las obras atendidas por la empresa y notificación de los resultados a empresa y directores de las obras.

Si la empresa se sirve de un centro de ensayos ajeno a ella, las misiones de prueba y control se efectuarán con contratos a largo plazo.

El centro permanente será dirigido por un técnico experimentado en la tecnología y fabricación del hormigón, que haya demostrado su formación y experiencia amplias mediante el certificado de un centro reconocido al efecto. La empresa únicamente empleará en obras con hormigón B II aquel personal directivo (directores de obras, capataces, etc.) que ya haya participado con responsabilidad en la fabricación, elaboración y tratamiento ulterior de hormigón de, por lo menos, la clase resistente Bn 250. El personal directivo de la obra y el personal técnico del centro permanente de ensayos se instruirán por la empresa, o bien por el jefe del centro permanente de ensayos, a intervalos de 3 años máximo, para asegurar la realización reglamentaria del proyecto de construcción.

\subsection{Prefabricados de hormigón}

Para la elaboración de prefabricados de hormigón que no se consideren producidos en fábrica, sirven las condiciones y requisitos para el hormigón en obra, conforme al criterio de la obra correspondiente. Prácticamente, no existen disposiciones especiales que difieran de lo determinado para hormigón en obra y para obras, a excepción de que para los prefabricados se exige siempre un control de calidad (apartado 3.3, apartado 4). En la elaboración de prefabricados de hormigón de los tipos resistentes $\mathrm{Bn} 50$ a Bn 250, ambos inclusive, se observarán las condiciones establecidas para hormigón B I (apartado 3.1), y en la preparación de prefabricados de hormigón de los tipos Bn 350 a Bn 550 se seguirán las condiciones dadas para hormigón B II (apartado 3.2).

Otro aspecto tiene la elaboración de prefabricados que se consideran producidos en fábrica. En estas fábricas puede producirse y utilizarse hormigón de todos los tipos resistentes. Para los tipos Bn 50 a Bn 250, ambos inclusive, la composición del hormigón se determinará como en el hormigón B I (apartado 3.1) y para los tipos Bn 350 a Bn 550 como en el hormigón B II (apartado 3.2), con la excepción de que en la prueba de idoneidad para los primeros no se ha fijado una medida determinada de predicción para la resistencia y tampoco es necesario cumplir la predicción de la consistencia (apartado 3.1 y DIN 1045, apartado 7.4.2.2). Además, para los tipos resistentes bajos ( $\mathrm{Bn} 50$ a $\mathrm{Bn} 250$ ), puede elegirse libremente la medida de predicción de la resistencia en la prueba de idoneidad. Ahora bien, debe ser tan amplia que al controlar la fabricación continua se cumplan con seguridad los requisitos exigidos [DIN 1084, hoja 2 (actualmente en proyecto)].

Los talleres de prefabricados de hormigón, cuyos productos deben considerarse como de fábrica, deben cumplir determinados requisitos (DIN 1045, apartado 5.3), incluso si estos talleres se montan provisionalmente, por ejemplo, como una "fábrica de campaña" en, o cerca, de una obra. Los talleres de prefabricados cumplirán, primordialmente, los requisitos en obras para hormigón B II. Por lo tanto, entre otras cosas, dispondrán de un centro de 
ensayos dirigido por un técnico experimentado en la tecnología y la fabricación de hormigón que aleccionará regularmente a su equipo técnico. Además estos talleres de prefabricados poseerán superficies de fabricación cubiertas para proteger contra climas desfavorables, recintos de fabricación y almacenamientos cerrados por todos los lados, posibilidad de calentamiento al hormigonar a temperaturas inferiores a $5^{\circ} \mathrm{C}$ y dispositivos para la protección contra climas adversos en el endurecimiento posterior del hormigón al aire libre. El jefe técnico del taller, o su representante competente, tienen que estar presentes durante la jornada de trabajo. Siempre que sea indicado, tiene iguales misiones que el director de las obras de la empresa y ha de procurar, asimismo, que se cumplan las condiciones exigidas al taller y que sólo salgan del mismo prefabricados según las normas con nota de entrega reglamentaria.

Con arreglo a DIN 1045, apartado 8, para todos los prefabricados de hormigón y de hormigón armado se efectuará un control de calidad, compuesto de control propio y control ajeno (véase también apartado 8). Como en el hormigón de obra, el volumen de los ensayos para hormigón de los tipos Bn 350 a Bn 550 es mayor que para el hormigón de los tipos $\mathrm{Bn} 50$ a Bn 250. Otros detalles de este control de calidad pueden tomarse de DIN 1084, hoja 2 (actualmente en proyecto, ver Betonstein Zeitung 36 (1970) n. ${ }^{\circ}$ 8).

En general, hay que observar que, para prefabricados de hormigón y de hormigón armado que poseen normas propias con dispositivos sobre calidad y ensayos, valen estas normas $y$, que también existen prefabricados que no están sometidos al control general de la obra y para los que tampoco hay normas establecidas. Para todos ellos valen las disposiciones de la DIN 1045, únicamente si se han observado estos criterios en las normas especiales o si se han convenido.

\subsection{Hormigón preamasado}

Para la preparación de hormigón preamasado sirven, fundamentalmente, las mismas disposiciones que para la confección del hormigón en obra, con la excepción de que para hormigón preamasado se exige siempre un control de la calidad.

Una regulación especial para el empleo del hormigón preamasado en la obra, como estaba todavía prevista en el proyecto amarillo de DIN 1045 (3), ya no es necesaria, puesto que en la nueva versión de DIN 1045 tanto el procedimiento de fabricación como la división en grupos B I y B II dependen del tipo resistente. Es decir, el hormigón preamasado Bn 50 hasta Bn 250, ambos inclusive, se fabrica como hormigón en obra B I (apartado 3.1) y puede suministrarse sin limitaciones a todas las obras de hormigón B I, y el hormigón preamasado Bn 350 a Bn 550 se fabrica como hormigón en obra B II (apartado 3.2) y puede suministrarse sin limitaciones a obras de este tipo de hormigón.

En las fábricas de hormigón preamasado puede prepararse hormigón según DIN 1045 de todos los tipos resistentes. A todas estas fábricas, aunque sólo se monten provisionalmente, se les exige determinadas condiciones con respecto a instalación, ocupación personal y funciones (DIN 1045, apartado 5.4), que corresponden a las del hormigón B II y prefabricados de hormigón. Claro es que también todas han de disponer, entre otras cosas, de un centro de ensayos dirigido por un técnico experimentado en la tecnología y la fabricación del hormigón y que adiestre regularmente a su personal.

Según DIN 1045, apartado 8, para el hormigón preamasado hay que efectuar un control de calidad, compuesto de control propio y control ajeno (véase también apartado 8). Co- 
mo en el hormigón en obra, el volumen de ensayos para el hormigón preamasado de los tipos Bn 350 a Bn 550 (hormigón B II) es mayor que para el de los tipos Bn 50 a Bn 250 (hormigón B I). Otros detalles de este control de calidad pueden tomarse de DIN 1084 hoja 3 (actualmente en proyecto), véase también apartado 8 .

\section{ADVERTENCIAS GENERALES SOBRE LA COMPOSICION DEL HORMIGON}

Además de los requisitos especiales en cuanto a la composición del hormigón (apartado 3), para los materiales de partida, el contenido de finos y la consistencia del hormigón fresco vale, en general, lo que se indica a continuación.

\subsection{Cemento}

Para todos los elementos resistentes de hormigón y hormigón armado según DIN 1045, únicamente debe emplearse cemento según DIN 1164 y/o un cemento equivalente autorizado. Solamente para hormigón sin armar del tipo Bn 50 están autorizados también los conglomerantes de mezcla, según DIN 4207.

\subsection{Arido para hormigón}

\subsubsection{Generalidades}

Como árido, para elementos resistentes de hormigón y hormigón armado según DIN 1045, únicamente se empleará el que cumpla los requisitos de DIN 4226. La nueva versión de DIN 4226, contiene todos los áridos, ya sean naturales o artificiales, triturados o no, así como los áridos con estructura compacta y porosa. En la refundición no sólo se tuvieron en cuenta los nuevos conocimientos, sino también todas las directrices actuales, por ejemplo, sobre escorias de alto horno, y piedra pómez siderúrgica, incluyendo los apartados sobre áridos ligeros de las Directrices de Hormigón Ligero Armado. La nueva DIN 4226 se compone de 3 hojas, o sea, hoja 1 para árido con estructura compacta, hoja 2 para árido con estructura porosa (árido ligero) y hoja 3 para todos los métodos de ensayo de los áridos reseñados en las hojas 1 y 2.

\subsubsection{Requisitos materiales}

Según DIN 4226, el árido cumplirá determinados requisitos de composición granulométrica, forma de grano, resistencia y consistencia y no contendrá cantidades perturbadoras de elementos nocivos. Los requisitos, sobre todo de resistencia, consistencia y porosidad, dependen de su aplicación y misión y, por lo tanto, pueden ser diferentes en cada caso. No obstante, el árido no se reblandecerá por la acción del agua, no se desintegrará, no formará combinaciones perturbadoras con los ingredientes del cemento y no se destruirá la protección anticorrosiva de la armadura en el hormigón. Se expresan con más precisión los requisitos y métodos de ensayo y se adaptan mejor a las circunstancias prácticas. Se incluyen nuevos métodos de ensayo para dictaminar la resistencia a las heladas del árido con los requisitos correspondientes, que se documentaron mediante múltiples análisis de numerosos áridos en varios Institutos. En el futuro, también los áridos para hormigón tendrán que someterse a un control de la calidad, compuesto de control propio y ajeno. 


\subsubsection{Composición granulométrica y grupos granulométricos}

Los cambios más importantes, tanto para el fabricante como para el consumidor, del árido para hormigón, afectan a la composición granulométrica global, los grupos granulométricos y su proporción en la mezcla. Para la composición granulométrica del árido sirven en la nueva norma curvas de tamizado ligeramente distintas, representadas en la figura 1 a 4 para mezclas de áridos de 8,16, 32 (tamaño nominal 32, criba de ensayo 31,5 $\mathrm{mm}$ ) y $63 \mathrm{~mm}$ de tamaño máximo. Según DIN 1045, los números de las zonas de cribado tienen en estas figuras el significado siguiente:

(1) Zona: desfavorable, realizable con demasiada dificultad.

(2) Zona: favorable para granulometrías discontinuas.

(3) Zona: favorable.

(4) Zona: utilizable, mayor necesidad de agua y cemento.

(5) Zona: desfavorable, consumo demasiado alto de agua y cemento.

Los tamaños de grano expresados en las figuras corresponden a la serie ISO, aprobada internacionalmente, y a la serie principal de DIN 66 100. En las cribas de ensayo ya no se utilizan agujeros redondos, sino cribas de mallas según DIN 4188 hasta inclusive $2 \mathrm{~mm}$ y cribas de agujeros cuadrados según DIN 4187, hoja 2. Para tener en cuenta la mayor importancia de la composición granulométrica en la zona de los finos y poder valorar mejor los valores acumulados, se hace una representación logarítmica eligiendo los tamices de forma que cada uno tenga el doble de luz que el siguiente. El tamaño máximo del árido para hormigón corriente, que hasta ahora era de $30 \mathrm{~mm}$ - referido a la criba de agujeros redondos-, será, en lo sucesivo, de $32 \mathrm{~mm}$, delimitado con la criba de agujero cuadrado de $31,5 \mathrm{~mm}$; esto corresponde, aproximadamente, a un tamaño máximo de $36 \mathrm{~mm}$ en la criba de agujeros redondos.

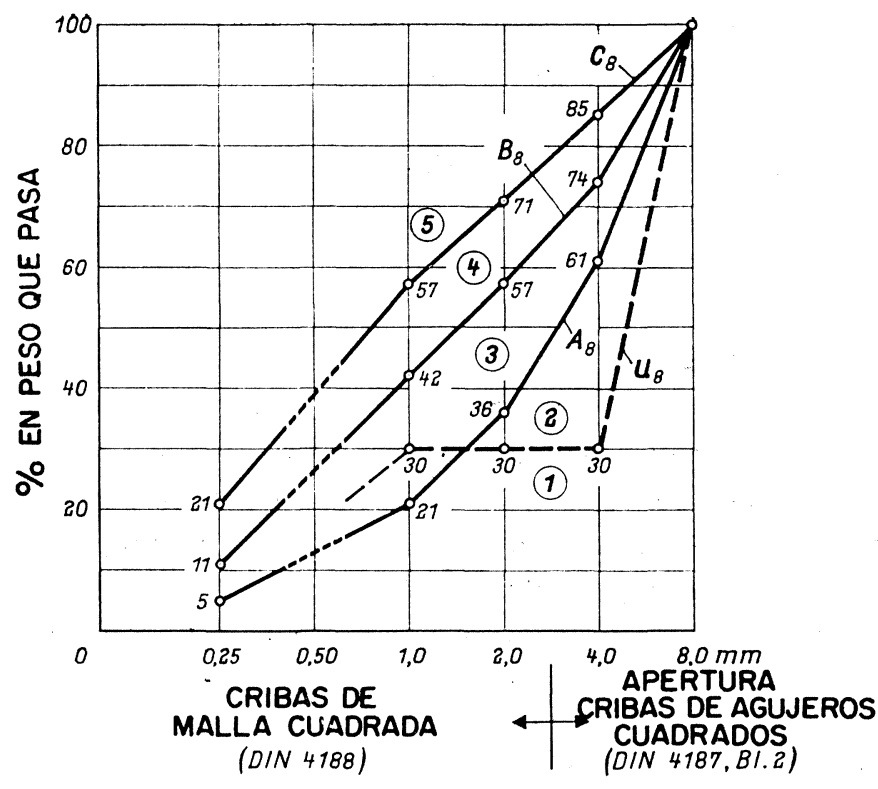

Fig. 1.-Curvas granulométricas límite para mezclas de árldos con un tamaño máximo de $8 \mathrm{~mm}$.

Las tres curvas A, B y C de la figura 3 corresponden en el fondo, en cuanto a la distribución proporcional a las curvas D, E y F de la norma antigua, con la excepción de que 
la curva $\mathbf{C}$ se bajó un poco en la zona de los finos con respecto a la curva F. Según DIN 1045, la composición granulométrica de mezclas de áridos con granulometría continua, para composiciones de hormigón, tabla 3, debe encontrarse, según el contenido mínimo de cemento, en la zona utilizable o en la favorable y, al establecer la composición del cemento, corresponder con la prueba determinante de idoneidad. En lo sucesivo, también se autorizan las granulometrías discontinuas para hormigones de todos los tipos resistentes en los que su composición se determina a base de una prueba de idoneidad. La línea de cribado U de las figuras 1 a 4 es la curva inferior límite para granulometrías discontinuas. Como modificación con respecto al proyecto amarillo de la DIN 1045, para adaptarse mejor a las condiciones naturales, se aumentaron los valores límite del residuo en $1 \mathrm{~mm}$, para las curvas B y C se aumentaron de 3 a $5 \%$, y los valores límite para la criba de 0,5 mm se eliminaron en todas las curvas, autorizándose también la granulometría discontinua para el hormigón B I con prueba de idoneidad.

Fig. 2.-Curvas granulométricas límite para mezclas de áridos con un tamaño máximo de $16 \mathrm{~mm}$.
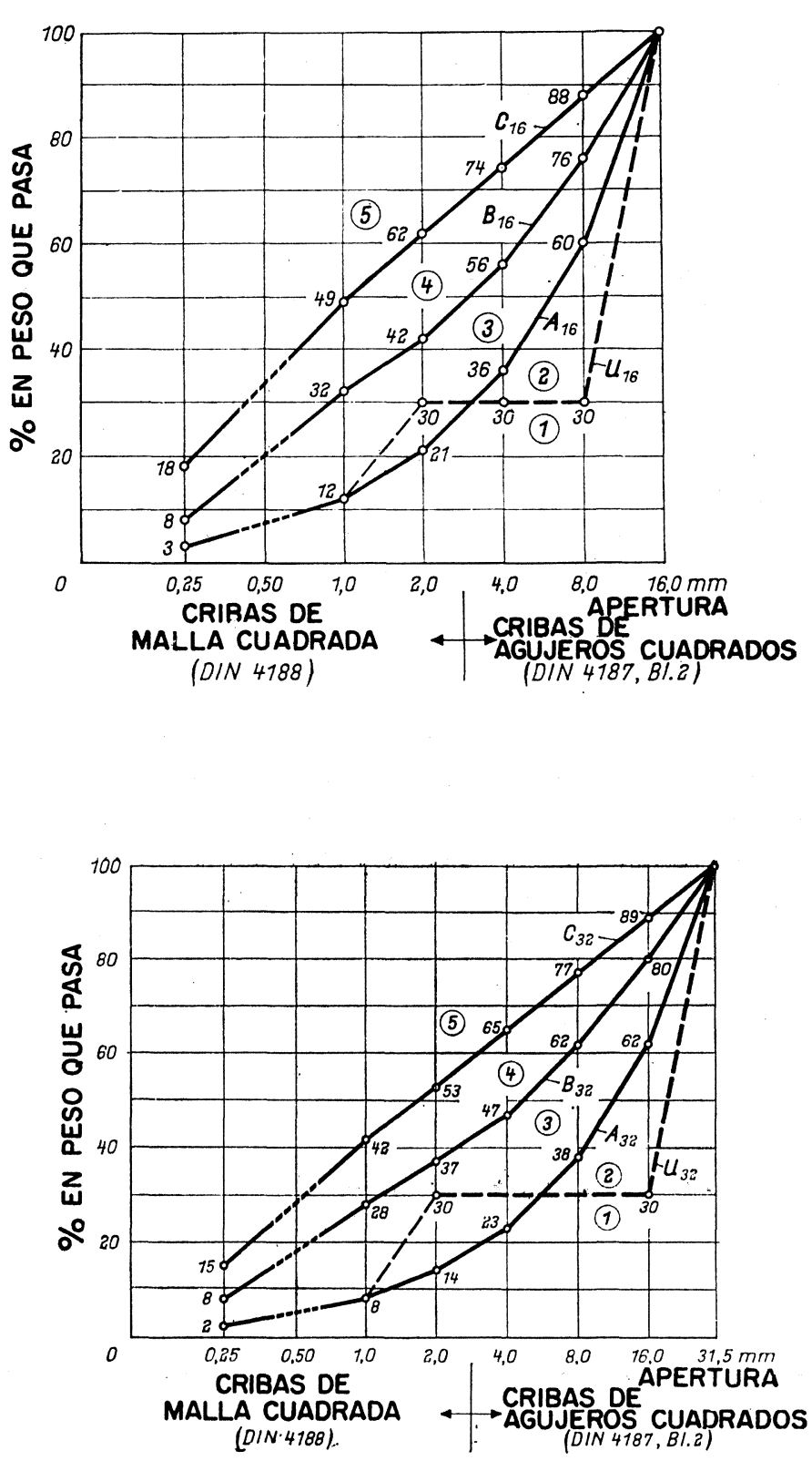

(DIN.4188)
Fig. 3.-Curvas granulométricas límite para mezclas de áridos con un tamano máximo de $32 \mathrm{~mm}$. 
En los ensayos se considera todavía como buena la composición granulométrica de mezclas de áridos, cuando el paso por las diversas cribas no difiere más del $5 \%$ del peso total de la curva establecida (en fracciones granulométricas con una compacidad distinta no deben diferir más del $5 \%$ de la compacidad del conjunto) y su valor característico para la composición granulométrica o la necesidad de agua no sea más desfavorable que en la curva fijada. En la fracción granulométrica $0 / 0,25$, las diferencias correspondientes sólo son tolerables hasta el $3 \%$.

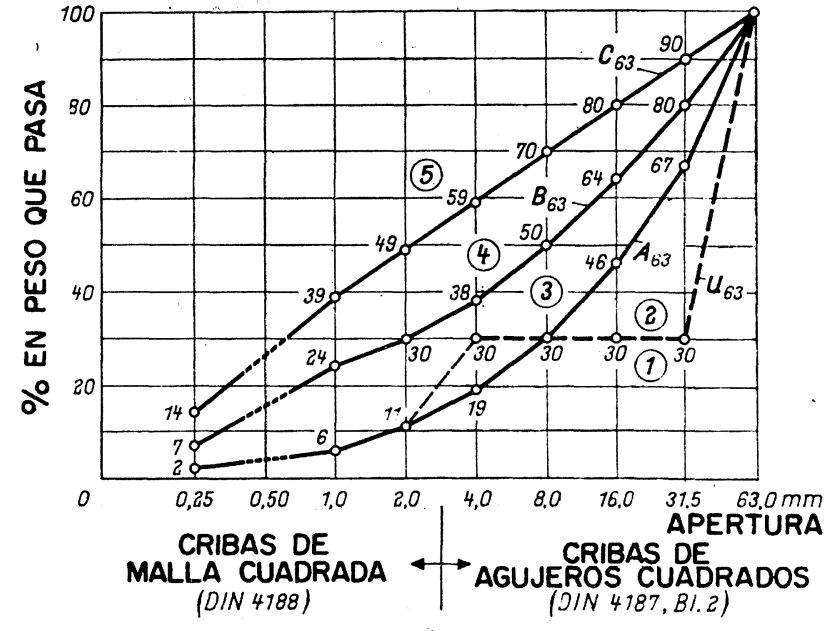

Fig. 4.-Curvas granulométricas límite para mezclas de áridos con un tamaño máximo de $63 \mathrm{~mm}$.

También, en lo sucesivo, hay que incorporar el árido en fracciones granulométricas separadas para todos los tipos resistentes de hormigón. Para hormigones B I y B II, con granulometría discontinua, hay previstas por lo menos dos fracciones granulométricas y para el hormigón B II, con composición granulométrica continua, se exigen, como mínimo, 3 fracciones granulométricas. De estas fracciones granulométricas, para el hormigón B I, una se encontrará, por lo menos, en la zona 0 a $4 \mathrm{~mm}$ y para el hormigón B II, por lo menos, en la zona de 0 a $2 \mathrm{~mm}$. Este desarrollo puede conducir, por lo tanto, para el hormigón B I a las fracciones granulométricas 0/4, 4/16 y 16/32 mm y para el hormigón B II a las fracciones granulométricas 0/2, 2/8, 8/16 y 16/32 mm. También será posible, emplear, en la preparación de hormigones de los tipos resistentes hasta un Bn 250 inclusive, en vez de fracciones granulométricas separadas, áridos mezclados en fábricas según DIN 4226, lo cual sólo puede hacerse con áridos de estructura compacta y un tamaño máximo de $32 \mathrm{~mm}$. También, para los áridos mezclados en fábrica, se permitieron granulometrías discontinuas, ya que se prohibió su almacenamiento intermedio en las estaciones de preparación de áridos (DIN 4226, hoja 1).

\subsection{Adiciones en el hormigón}

\subsubsection{Aditivos}

Por aditivos al hormigón se entienden aquellas sustancias que se agregan al mismo en cantidades pequeñas como, por ejemplo, oclusores de aire, fluidificantes y retardadores, 
que no repercuten en el volumen del hormigón. Según la nueva versión de la DIN 1045, para elementos de hormigón y hormigón armado sólo se permitirá el empleo de aditivos con ensayos garantizados, es decir, sustancias que cumplan las directrices establecidas para los aditivos (7). Estas son sustancias que no forman combinaciones perturbadoras con los ingredientes del hormigón y que no degeneran en la protección anticorrosiva de las armaduras. Pero como quiera que los ensayos de garantía no dicen nada sobre el modo de actuar, porque, por ejemplo, al incorporar un aireante no sabemos si se ha formado un número suficiente de microporos, el usuario puede comprobar en la prueba de la idoneidad, y antes de su empleo el consumidor debe exigir al fabricante del aditivo certificados de un Centro reconocido al efecto sobre el modo de actuar. Además, como en cada caso individual tanto los componentes como la composición del hormigón y las demás condiciones de su fabricación son de importancia, al realizar una fabricación continua de hormigón empleando aditivos, la composición final del hormigón se establecerá siempre con ayuda de una prueba de idoneidad.

\subsubsection{Adiciones}

Por adiciones, la DIN 1045 considera aquellas sustancias finamente divididas que se agregan al hormigón en cantidad mayor, repercuten en su volumen y poseen algunas propiedades hidráulicas latentes. Las adiciones no deben perturbar el endurecimiento del cemento, la resistencia y consistencia del hormigón y la protección anticorrosiva de la armadura. En caso de duda se hará la prueba de idoneidad. Como quiera que en el pasado sólo se trataba de sustancias minerales finas, hasta ahora podían agregarse al hormigón sin más hasta llegar al contenido necesario de finos o para mejorar la elaborabilidad. Sin embargo, puede añadirse al cemento una sustancia hidráulica latente o puzolánica solamente si está establecido de un modo especial, como por ejemplo por una autorización general del control de la obra. Adiciones extrañas, plásticos por ejemplo, necesitan, en cada caso, una autorización a base de una demostración de la idoneidad con arreglo a las directrices establecidas para ello, y antes de emplearlas en el hormigón, de una prueba de idoneidad hecha por el consumidor.

\subsection{Contenido de finos}

Como finos se consideran todas las fracciones granulométricas del hormigón hasta 0,25 $\mathrm{mm}$, es decir, el cemento, la fracción de 0/0,25 contenida en el árido y, en su caso, un aditivo o una adición comprendidos en este área granulométrica. El hormigón tiene que contener una cantidad determinada de finos, a fin de que se pueda elaborar correctamente y obtener una estructura cerrada. Los valores de orientación de la DIN 1045 para el contenido de finos se encuentran en la tabla 4. Las zonas citadas en el proyecto amarillo para el contenido de finos se sustituyeron por un valor de orientación, ya que la proporción de finos en los recursos naturales era muy distinta al pasar de la criba de 0,2 a la de $0,25 \mathrm{~mm}$, y por eso no se pueden indicar en forma sencilla zonas válidas para todos los casos. Pero como quiera que un exceso de finos puede menoscabar determinadas propiedades del hormigón, tales como la resistencia a las heladas, la resistencia a las sales de deshielo y la resistencia a los ataques químicos, junto con los valores de orientación para el contenido de finos se incluyó en DIN 1045 una advertencia de que el contenido de finos, sobre todo en relación con las propiedades del hormigón según DIN 1045, apartado 6.5.7.3 a 6.5.7.5, hay que limitarlo lo más posible a lo necesario para la elaborabilidad, ya que 
una proporción demasiado elevada puede repercutir desfavorablemente en las citadas propiedades.

T A B L A 4

Valores de orientación para el contenido de finos según DIN 1045

(sustancias finas $\leqslant 0,25 \mathrm{~mm}$ )

\begin{tabular}{|c|c|}
\hline Tamaño máximo de la mezcla de áridos $(\mathbf{m m})$ & $\begin{array}{c}\text { Valores de orientación *) para el contenido } \\
\text { de finos en kg por cada } \mathbf{m}^{3} \text { de hormigón } \\
\text { compactado }\end{array}$ \\
\hline 8 & 525 \\
16 & 450 \\
63 & 400 \\
& 325 \\
\hline
\end{tabular}

*) A ser posible limitación con propiedades del hormigón según DIN 1045, apartado 6.5.7.3 a 6.5.7.5.

\subsection{Consistencia del hormigón fresco}

La consistencia es una medida de la trabajabilidad del hormigón fresco. En la DIN 1045 antigua se diferenciaban 3 tipos de consistencia: seca, plástica y fluida. En la nueva versión de DIN 1045 ya no existe el hormigón fluido, puesto que se segrega, y, en determinadas circunstancias, su utilización puede crear peligros para la seguridad de la obra, aparte de que con las actuales posibilidades de compactación no es necesario su empleo en las construcciones de hormigón, salvo casos especiales. En la nueva DIN 1045 también se distinguen tres tipos de consistencia (tabla 5), ya que para el hormigón vibrado propiamente dicho se requiere un tipo de consistencia $\mathrm{K} 2$, comprendido entre las consistencias rígida (K 1) y plástica (K 3). En cada caso, la consistencia se determinará con precisión mediante medida del asentamiento o del escurrimiento del hormigón (tabla 5 y DIN 1048).

T A B L A 5

Consistencia del hormigón según DIN 1045

\begin{tabular}{|c|c|c|c|c|c|}
\hline \multirow{2}{*}{\multicolumn{2}{|c|}{ Gama de consistencias }} & \multicolumn{2}{|c|}{ Propiedades del } & \multirow{3}{*}{$\begin{array}{l}\begin{array}{l}\text { Medida de la } \\
\text { compactación }\end{array} \\
1,45 \text { a } 1,26\end{array}$} & \multirow{3}{*}{$\begin{array}{r}\begin{array}{r}\text { Dimensiones } \\
\text { de una torta } \\
\text { de hormigón }\end{array} \\
-\end{array}$} \\
\hline & & \multirow{2}{*}{$\begin{array}{c}\text { Mortero fino } \\
\begin{array}{c}\text { Algo más blando que } \\
\text { la tierra húmeda }\end{array}\end{array}$} & \multirow{2}{*}{$\begin{array}{c}\begin{array}{c}\text { Hormigón fresco } \\
\text { al verterlo }\end{array} \\
\text { Todavía suelto }\end{array}$} & & \\
\hline K 1 & Hormigón seco & & & & \\
\hline K 2 & Hormigón plástico & Blando & $\begin{array}{l}\text { Aterronado hasta } \\
\text { escasamente } \\
\text { coherente }\end{array}$ & 1,25 a 1,11 & $\leqslant 40$ \\
\hline K 3 & Hormigón fluido & Fluido & $\begin{array}{l}\text { Fluyendo } \\
\text { lentamente }\end{array}$ & 1,10 a 1,04 & 41 a 50 \\
\hline
\end{tabular}

Para una mejor coincidencia de ambos métodos de ensayo, se modificaron algo los valores límite de los tipos de consistencia frente al proyecto amarillo de la DIN 1045. No obs- 
tante, se observa que no puede existir una coincidencia completa entre ambos métodos de medida de la consistencia, porque la forma de actuar en ellos es distinta y así, por ejemplo, al producirse ciertos cambios en la composición del hormigón (mayor tamaño máximo del árido, árido triturado, granulometría discontinua) no se comportan de igual modo. En casos especiales puede, por lo tanto, ser conveniente establecer uno de los dos métodos para delimitar la consistencia.

\section{PROTECCION ANTICORROSIVA DE LA ARMADURA}

Una condición previa, esencial en el hormigón armado, es que la armadura, que sin protección se corroería al aire muy rápidamente, empotrada en el hormigón queda protegida contra corrosión. Esto queda asegurado cuando el hormigón que rodea el acero es compacto, de suficiente espesor y no contiene aditivos que menoscaben la protección anticorrosiva de la armadura en el hormigón, tales como cloruros. La nueva versión de DIN 1045 tiene esto en cuenta y exige para hormigón armado, además de la prohibición en el uso de aditivos que activen la corrosión, que en el hormigón el contenido de cemento 250 según DIN 1164 no sea menor de $280 \mathrm{~kg} / \mathrm{m}^{3}$ y no se sobrepase la relación agua/cemento de 0,65. Esto son condiciones complementarias, bien establecidas para crear una protección suficiente de la armadura, ya que por otros motivos, por ejemplo para obtener una determinada resistencia o para conseguir propiedades especiales (apartado 6), pueden ser necesarios contenidos de cemento mayores y relaciones agua/cemento menores. Estos valores límite para el contenido de cemento y la relación agua/cemento hay que observarlos en todos los hormigones cuya composición se establece en virtud de una prueba de idoneidad. Sin embargo, en hormigón de los tipos resistentes Bn 250 y superiores prácticamente no hay limitaciones. En el procedimiento de preparación del hormigón B I según apartado 3.1 , párrafo 1 , con contenidos mínimos de cemento según tabla 3 , ya se tuvieron en cuenta estas condiciones.

$$
\text { T А в L A } 6
$$

Espesor mínimo del recubrimiento de la armadura en función de las condiciones del medio ambiente

\begin{tabular}{|c|c|c|c|c|c|}
\hline \multirow{4}{*}{$\begin{array}{l}\text { Caracterización del } \\
\text { ambiente } \\
\text { alrededor del elemento }\end{array}$} & \multicolumn{5}{|c|}{ Espesor mínimo del recubrimiento de la armadura en $\mathrm{cm}$ con } \\
\hline & \multicolumn{4}{|c|}{ Hormigón in situ $y$ prefabricados } & \multirow{3}{*}{$\begin{array}{l}\text { Prefabricados } \\
\text { producidos } \\
\text { en fábrica } \\
\geqslant \text { Bn } 350\end{array}$} \\
\hline & \multicolumn{2}{|c|}{ Bn 150} & \multicolumn{2}{|c|}{$\geqslant$ Bn 250} & \\
\hline & General & $\begin{array}{l}\text { Superficies } \\
\text { planas } * \text { ) }\end{array}$ & General & $\begin{array}{l}\text { Superficies } \\
\text { planas } * \text { ) }\end{array}$ & \\
\hline $\begin{array}{l}\text { Locales cerrados } \\
\text { normales }\end{array}$ & 2,0 & 1,5 & 1,5 & 1,0 & 1,0 \\
\hline $\begin{array}{l}\text { Entrada del aire } \\
\text { exterior }\end{array}$ & $\mathbf{2 , 5}$ & 2,0 & 2,0 & 1,5 & 1,5 \\
\hline $\begin{array}{l}\text { Fomentando débilmen- } \\
\text { te la corrosión o ata- } \\
\text { cando }{ }^{* *} \text { ) débilmente } \\
\text { al hormigón según } \\
\text { DIN } 4030\end{array}$ & 3,0 & 2,5 & 2,5 & 2,0 & 2,0 \\
\hline $\begin{array}{l}\text { Fomentado intens a- } \\
\text { mente la corrosión o } \\
\text { atacando fuertemente } \\
\text { al hormigón según } \\
\text { DIN } 4030\end{array}$ & 4,0 & 3,5 & 3,5 & 3,0 & 3,0 \\
\hline
\end{tabular}

*) Estructuras planas, según estas tablas, son placas (también las placas nervadas), forjados de hormigón aligerado, láminas, cáscaras, estructuras plegadas y paredes.

**) También en locales con humedad ambiente alta pero variable. 
Los requisitos para el espesor mínimo de recubrimiento de la armadura se supeditan a las condiciones del medio ambiente y al diámetro de la armadura. Las disposiciones más esenciales de la nueva DIN 1045 se deducen de las tablas 6 y 7 . El valor determinante es el máximo de las dos tablas, siempre que no se indique un aumento del espesor del hormigón por otros motivos o sea conveniente en un caso individual, pues los valores de las citadas tablas 6 y 7 sólo son valores mínimos. Para hormigón armado corriente, el espesor mínimo de hormigón sobre la armadura es, en lo sucesivo, por término medio, del orden de $0,5 \mathrm{~cm}$ mayor que hasta ahora.

T A в L A 7

Recubrimiento mínimo de hormigón en la armadura en función del diámetro de las varillas (barras)

\begin{tabular}{|c|c|}
\hline Diámetro de las barras (mm) & Recubrimiento mínimo de hormigón (mm) \\
\hline 14 & 15 \\
16 & 15 \\
18 & 15 \\
\hline 20 & -20 \\
22 & 20 \\
\hline 25 & 25 \\
\hline 28 & 30 \\
\hline Por encima de 28 \\
\hline
\end{tabular}

\section{HORMIGON CON PROPIEDADES ESPECIALES}

Puesto que los hormigones con propiedades especiales se utilizan cada vez más, en la nueva versión de DIN 1045 se incluyen también indicaciones para: hormigón impermeable al agua, hormigón resistente a las heladas, hormigón con resistencia a los ataques químicos, hormigón resistente al desgaste (tabla 8), hormigón con suficiente resistencia

T А в L A 8

Condiciones de la DIN 1045 para impermeabilidad al agua, elevada resistencia a las heladas y elevada resistencia al desgaste

\begin{tabular}{|c|c|}
\hline Impermeabilidad al agua & $\begin{array}{c}\text { Profundidad máxima de penetración del agua } \\
\text { según DIN } \mathbf{1 0 4 8} \mathbf{e}_{\max } \leqslant \mathbf{5} \text { cm relación } \\
\text { agua/cemento } \leqslant \mathbf{0 , 6 0}\end{array}$ \\
\hline Elevada resistencia & $\begin{array}{c}\text { Hormigón impermeable al agua o relación } \\
\text { agua/cemento } \leqslant 0,70 \mathrm{y} \text { adición de aire } \\
\text { ocluido }\end{array}$ \\
\hline $\begin{array}{c}\text { Elevada resistencia } \\
\text { al desgaste }\end{array}$ & $\begin{array}{c}\text { Con acción de las sales de deshielo - pres- } \\
\text { cindiendo del hormigón muy seco- siem- } \\
\text { pre adición de aire ocluido }\end{array}$ \\
\hline contenido de cemento $\leqslant 350 \mathrm{~kg} / \mathrm{m}^{3}$ \\
\hline
\end{tabular}


al calor, y hormigón subacuático. Condición previa para el logro de las condiciones especiales es que el hormigón se componga, fabrique y coloque convenientemente, que no se segregue, que se compacte totalmente y su tratamiento ulterior sea suficiente, ya que todas las propiedades especiales exigen un hormigón y una pasta endurecida lo más compactos posible. Para la fabricación de hormigón con propiedades especiales rigen fundamentalmente las condiciones para hormigón B II, siempre que no se advierta expresamente la validez de las condiciones para hormigón B I.

\subsection{Hormigón impermeable al agua}

Con arreglo a la nueva versión de DIN 1045, el hormigón impermeable al agua para elementos con un espesor de 10 a $40 \mathrm{~cm}$ cumplirá las condiciones indicadas en la tabla 8. Por una experiencia de décadas mediante ensayos y observación del comportamiento práctico del hormigón correspondiente, se sabe que el hormigón para los elementos antes mencionados es prácticamente impermeable al agua en su aplicación cuando la máxima profundidad de penetración del agua (media de 3 valores), al ensayarlo según DIN 1048, no excede de $5 \mathrm{~cm}$ y su relación agua/cemento no sobrepasa 0,60. Esto significa que teniendo en cuenta las dispersiones habituales en la fabricación continua de hormigón hay que aspirar, en general, a una relación agua/cemento de 0,55 como máximo. Con elementos más gruesos que $40 \mathrm{~cm}$ no debe sobrepasarse la relación agua/cemento 0,70 .

En proyectos de obras menores puede fabricarse un hormigón B II impermeable al agua, de resistencia menor que $\mathrm{Bn} 350$, en las mismas condiciones que para el hormigón $\mathrm{B}$ I, cuando el contenido de cemento con áridos $0 / 16 \mathrm{~mm}$ es, por lo menos, de $400 \mathrm{~kg} / \mathrm{m}^{3}$ y con áridos $0 / 32 \mathrm{~mm}$ de, por lo menos, $350 \mathrm{~kg} / \mathrm{m}^{3}$, siempre que la composición granưlométrica de los áridos se encuentre en la zona favorable de las figuras 2 ó 3.

Fundamentalmente hay que observar que esto son sólo las condiciones para el material de construcción "hormigón impermeable al agua". En la fabricación de elementos de construcción impermeables al agua se procurará, adoptando las medidas necesarias en la ejecución de la obra y constructivas, que estos elementos no acusen ningún defecto que elimine la impermeabilidad al agua, tales como grietas y juntas no estancas.

\subsection{Hormigón resistente a las heladas}

Los requisitos complementarios de la nueva versión de DIN 1045 para hormigón de resistencia a las heladas y resistente a las sales de deshielo se deducen de las tablas 8 y 9. Por numerosos ensayos y amplias experiencias prácticas se sabe que el hormigón impermeable al agua con relación agua/cemento $\leqslant 0,60$ (apartado 6.1) manifiesta en estado de saturación una elevada resistencia, suficiente para las condiciones climatológicas habituales en Alemania, a cambios frecuentes y bruscos de hielo-deshielo. Para la fabricación de tales hormigones se utilizarán áridos con alta resistencia a las heladas con "acción intensa de las heladas" según DIN 4226. En elementos de construcción voluminosos la relación agua/cemento puede ser de hasta 0,70 , si por adición de un oclusor de aire adecuado (apartado 4.3.1) se produce suficiente número de pequeñas burbujas de aire y si los elementos de construcción no entran en contacto con sales de deshielo. El contenido necesario de aire ocluido depende de la cantidad de mortero fino (cemento, agua y sustancias finas hasta $0,25 \mathrm{~mm}$ ) [véase entre otros (8)]. Para simplificar, en el ensayo con 
hormigón fresco se determinó el contenido de aire en función del tamaño máximo del árido y no deberá quedar por debajo de los valores de la tabla 9.

T A в L A 9

Contenido de aire en el hormigón fresco

\begin{tabular}{|c|c|}
\hline Tamaño máximo del árido $(\mathrm{mm})$ & Contenido medio de aire $*$ ) $\%$ de volumen \\
\hline 8 & $\geqslant 5,0$ \\
16 & $\geqslant 4,0$ \\
32 & $\geqslant 3,5$ \\
63 & $\geqslant 3,0$ \\
\hline
\end{tabular}

*) Los valores particulares quedarán, como máximo, 0,5 \% por debajo de esta exigencia.

El contenido de aire ocluido indicado en la tabla 9 -aparte de en hormigones muy secos- es indispensable también para relaciones agua/cemento menores, si el hormigón entra frecuentemente en contacto con sales de deshielo y está sometido a ciclos de hielodeshielo.

Con iguales condiciones previas que en el hormigón impermeable al agua, el hormigón con alta resistencia a las heladas y tipo resistente inferior $\mathrm{Bn} 350$ puede fabricarse también en las condiciones del hormigón $\mathrm{B}$ I.

\subsection{Hormigón con gran resistencia a los ataques químicos}

\subsubsection{Grado de ataque}

A fin de dictaminar el grado de ataque de aguas, suelos y gases al hormigón y los correspondientes métodos de análisis, se refundió la DIN 4030 y también se publicó como nueva edición en noviembre 1969. Las aguas sospechosas se conocen frecuentemente por sus características exteriores, es decir, por su color, olor, subida de burbujas de gas, precipitación de sales y coloración roja del papel de tornasol. El grado de ataque de aguas de composición preferentemente natural se dictaminará en lo sucesivo con los valores límite de la tabla 10 , si no se excede de un consumo de permanganato potásico de $50 \mathrm{mg} / \mathrm{li}$ -

T A B L A 10

Dictamen del grado de ataque de las aguas según DIN 4030

\begin{tabular}{|c|c|c|c|}
\hline \multirow{2}{*}{ Compuestos agresivos } & \multicolumn{3}{|c|}{ Grado de ataque } \\
\hline & Ataque débil & Ataque fuerte & Ataque muy fuerte \\
\hline Valor del pH (ácidos) & 6,5 a 5,5 & 5,5 a 4,5 & por debajo de 4,5 \\
\hline $\begin{array}{l}\text { Acido carbónico que } \\
\text { disuelve la cal } \mathrm{CO}_{2} \\
\text { en } \mathrm{mg} / l \text { (ensayo del } \\
\text { mármol según Heyer) }\end{array}$ & 15 a 30 & $30 \mathrm{a}$ & por encima de \\
\hline Amonio $\mathrm{NH}_{4}^{+}$en $\mathrm{mg} / l$ & 15 a 30 & $30 \mathrm{a}$ & por encima de \\
\hline Magnesio $\mathrm{Mg}^{2+}$ en $\mathrm{mg} / \mathrm{l}$ & 100 a 300 & 300 a 1.500 & por encima de 1.500 \\
\hline Sulfato $\mathrm{SO}_{4}{ }^{2-}$ en $\mathrm{mg} / \mathrm{l}$ & 200 a 600 & 600 a 3.000 & por encima de 3.000 \\
\hline
\end{tabular}


tro y las características exteriores no indican otras sustancias atacantes. Los valores límite de la tabla 10 tienen en cuenta las diversas sustancias, diferencian tres grados de ataque, son fáciles de aplicar y se eligieron de modo que los grados de ataque "débil" e "intensamente agresiva" puede soportarlos un hormigón de composición conveniente. Los valores límite sirven para aguas estancas y de corriente débil, existentes en gran cantidad y que atacan directamente. El grado de ataque en cada caso aumenta un escalón cuando dos o más valores se hallan en el límite superior (con el valor $\mathrm{pH}$ en el límite inferior). Pero esto no sirve, sin embargo, para agua de mar, ya que, según la experiencia, un hormigón suficientemente compacto, expuesto desde hace muchos años, opone la suficiente resistencia al agua marina. La capacidad de ataque del agua puede aumentar por corrientes fuertes, temperatura más elevada y presión más alta, y disminuye con una permeabilidad decreciente del terreno.

T A B L A 11

Dictamen del grado de ataque de suelos según DIN 4030

\begin{tabular}{|l|c|c|}
\hline \multicolumn{1}{|c|}{ Sustancias agresivas } & Ataque débil & Ataque fuerte \\
\cline { 2 - 3 } & por encima de 20 \\
$\begin{array}{l}\text { Acidos } \\
\text { grado de acidez según } \\
\text { Baumann-Gully }\end{array}$ & 2.000 a 5.000 & por encima de 5.000 \\
\hline $\begin{array}{l}\text { Sulfato } \\
\text { en mg/kg suelo seco al aire }\end{array}$ & \\
\hline
\end{tabular}

Para el dictamen de la capacidad de ataque de suelos naturales, que frecuentemente se empapan pero que no permiten tomar una muestra de agua, la norma DIN 4030 establece los valores límite de la tabla 11. En terraplenes, en terrenos con residuos industriales o en presencia de sulfuros, se necesita en general un análisis posterior. Existiendo gases residuales industriales con mayor concentración, como, por ejemplo, en cámaras de filtrado, chimeneas de gases de escape, etc, puede ser necesario un dictamen pericial.

\subsubsection{Medidas tecnológicas del hormigón}

Las medidas a tomar en caso de ataques químicos según DIN 4030 están contenidas en la nueva versión de DIN 1045; las medidas complementarias de la tecnología del hormigón de la DIN 1045 se reproducen en la tabla 12. Ataques "débiles" según DIN 4030 los resiste bastante bien un hormigón impermeable al agua con relación agua/cemento $\leqslant 0,60$ (apartado 6.1). Bajo iguales condiciones previas que en el hormigón impermeable al agua, el hormigón con resistencia al ataque químico "débil" y tipo resistente inferior al Bn 350 puede fabricarse también en las condiciones dadas para el hormigón B I.

En el hormigón con resistencia a ataques químicos "intensos", la máxima profundidad de penetración del agua según DIN 1048 no debe exceder de $3 \mathrm{~cm}$ y la relación agua/cemento de 0,50. Como consecuencia de las inevitables dispersiones en la fabricación continua del hormigón, debe aspirarse a una relación agua/cemento de 0,45 como máximo. El hormigón no puede exponerse durante un tiempo prolongado a ataques químicos "muy inten- 
sos"; en tales casos, además de un hormigón con resistencia a los ataques químicos "intensos", es siempre necesaria una protección duradera del hormigón.

T A B L A 12

Exigencias al hormigón con elevada resistencia contra los ataques químicos según DIN 4030

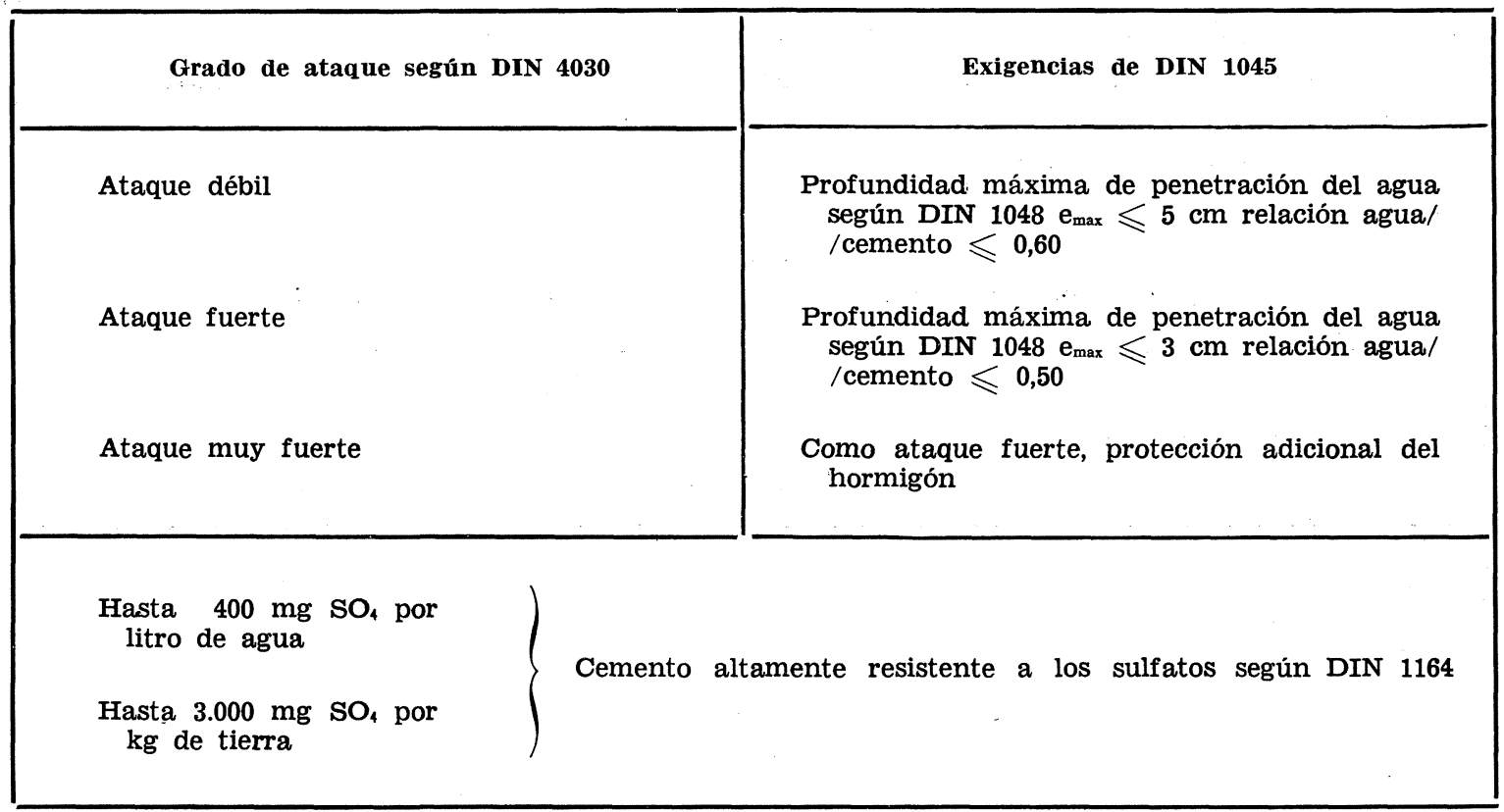

También bajo una acción permanente intensa de gases que atacan al hormigón (como pueden ser ciertos gases residuales industriales) o de sustancias que atacan al acero (por ejemplo aire con gran contenido de agua de mar o aguas con más de $1.500 \mathrm{mg}$ de cloro por litro), debe utilizarse un hormigón resistente a ataques "débiles" (tabla 12). La composición del hormigón debe cumplir los requisitos para ataque "intenso" (tabla 12), si los gases que atacan al hormigón actúan durante largo tiempo con concentración fuerte y/o locales limitados, si es posible que se llegue al punto de rocío (por ejemplo en cámaras de filtrado o en chimeneas de gases de escape de determinadas industrias) o si con frecuencia o durante largo tiempo afectan al hormigón soluciones muy concentradas de cloruros.

Independientemente del grado de ataque existente en cada caso, para agua de mar, con contenidos en sulfatos superiores de $400 \mathrm{mg} \mathrm{SO}{ }_{4}$ por litro y terrenos con más de $3.000 \mathrm{mg}$ $\mathrm{SO}_{4}$ por kg, además de un hormigón compacto, se empleará un cemento con alta resistencia a los sulfatos según DIN 1164. Estos son: cementos portland con un contenido calculado de aluminato tricálcico $\mathrm{C}_{3} \mathrm{~A}$ de $3 \%$ en peso máximo y un contenido de óxido de aluminio de $5 \%$ en peso máximo y cementos de horno alto con un mínimo de $70 \%$ en peso de escoria granulada y $30 \%$ máximo de clínker de cemento portland.

Más indicaciones sobre medidas constructivas, ejecución, recubrimiento de armaduras y protección del hormigón contra los ataques químicos, se encontrarán en otro lugar [véase entre otros (9)]. 


\section{\%. EJECUCION DE LA CONSTRUCCION}

Este apartado ejecución de la construcción, que trata de la fabricación, transporte, compactación y tratamiento posterior del hormigón, hormigonado a bajas temperaturas y bajo agua, así como los temas de juntas de dilatación, encofrado y colocación de armaduras, y que sólo en parte puede aplicarse a prefabricados de hormigón, fue asimismo adaptado al estado del progreso. Con respecto a las modificaciones de importancia tecnológica hay que citar especialmente lo siguiente:

Cemento, árido y agua se aplicarán en lo sucesivo con una precisión del $3 \%$ en peso. Como duración mínima del amasado, para amasadoras con rendimiento especialmente bueno (DIN 459), se exigen 0,5 minutos, y a todas las demás 1 minuto, después de añadir todos los ingredientes. En hormigón con textura cerrada hay que aspirar siempre a una compactación prácticamente total. $\mathrm{Al}$ hormigón hay que protegerlo normalmente un mínimo de 7 días contra la desecación. Para el tratamiento posterior, en lugar de mantenerlo húmedo, se permite la aplicación de películas de tratamiento y, en prefabricados, un tratamiento térmico correcto en lo que se refiere a la tecnología del hormigón [véase entre otros (10)]. Cuando existe tratamiento térmico es indispensable una prueba de idoneidad en las condiciones correspondientes.

T A в L A 13

Valores de orientación para plazos de desencofrado

\begin{tabular}{|c|c|c|c|}
\hline $\begin{array}{l}\text { Tipo resistente del ce- } \\
\text { mento según DIN } 1164\end{array}$ & $\begin{array}{l}\text { Encofrado de paredes, } \\
\text { soportes y vigas } \\
\text { (lateralmente) } \\
\text { (días) }\end{array}$ & $\begin{array}{c}\text { Encofrado de placas } \\
\text { de cubierta } \\
\text { (días) }\end{array}$ & $\begin{array}{l}\text { Armadura de vigas, } \\
\text { marcos y placas de } \\
\text { luz grande } \\
\text { (días) }\end{array}$ \\
\hline 250 & 4 & 10 & 28 \\
\hline $350 \mathrm{~L}$ & 3 & 8 & 20 \\
\hline $\begin{array}{c}350 \mathrm{~F} \\
y \\
450 \mathrm{~L}\end{array}$ & 2 & 5 & 10 \\
\hline $\begin{array}{c}450 \\
y \\
550\end{array}$ & 1 & 3 & 6 \\
\hline
\end{tabular}

Las medidas a tomar para el hormigonado a bajas temperaturas se ajustaron a las directrices de la construcción en invierno. Se incluyó también la advertencia de que un hormigón joven con un contenido de cemento de por lo menos $270 \mathrm{~kg} / \mathrm{m}^{3}$ y una relación agua/cemento de 0,60 como máximo no puede helarse, si su temperatura, empleando cementos de endurecimiento rápido, no baja de $10^{\circ} \mathrm{C}$ durante 3 días o si ha alcanzado ya una resistencia a la compresión de $50 \mathrm{kp} / \mathrm{cm}^{2}$ como mínimo.

La nueva versión de la DIN 1045 contiene, como valores de orientación a temperaturas de endurecimiento de más de $5^{\circ} \mathrm{C}$, los plazos de desencofrado de la tabla 13 , que, sin embargo, únicamente tienen un carácter de aproximación, ya que el desarrollo del endurecimiento depende de numerosos factores de influencia. Determinante para el desencofrado es, por lo tanto, la resistencia lograda en el caso particular. El director de las obras puede ordenar el desencofrado cuando ya se ha convencido plenamente de la suficiente resistencia del hormigón (prueba del endurecimiento). 


\section{COMPROBACION Y CONTROL DE LA CALIDAD}

Los datos sobre el tipo y volumen de los ensayos a realizar en cada caso particular y sobre los requisitos a cumplir, se encuentran: para el hormigón B I en DIN 1045, apartado 7, y. para el hormigón B II en DIN 1045, apartado 7, y. DIN 1084, hoja 1 (actualmente en proyecto), para prefabricados de hormigón en DIN 1084, hoja 2 (actualmente en proyecto), y para hormigón preamasado en DIN 1084, hoja 3 (actualmente en proyecto). Ya no se exige una comprobación de los materiales de partida ni en la obra, ni en la fábrica de prefabricados de hormigón ni en la estación de hormigón preamasado. Unicamente, la composición granulométrica de los áridos hay que comprobarla en la primera entrega, al cambiarse de proveedor $\mathrm{y}$, además, a intervalos prudenciales; es decir, siempre con hormigón B II y con hormigón B I, solamente si la composición granulométrica se determinó en virtud de una prueba de idoneidad o tiene que corresponder a la tabla 3 con composición granulométrica favorable.

T A B L A 14

Volumen de los ensayos de calidad según DIN 1045 para cada tipo de hormigón fabricado

\begin{tabular}{|c|c|}
\hline Hormigón B I & Hormigón B II \\
\hline Consistencia: Continua a simple vista. & \\
\hline $\begin{array}{l}\text { Medida de consistencia: En la } 1 .{ }^{\mathrm{a}} \text { colocación } \\
\text { del hormigón y al confeccionar las probetas } \\
\text { de resistencia. }\end{array}$ & $\begin{array}{l}\text { Consistencia: Medida de consistencia y con- } \\
\text { tenido de cemento como en hormigón B I. }\end{array}$ \\
\hline $\begin{array}{l}\text { Contenido de cemento: En la } 1 .^{\text {a }} \text { colocación } \\
\text { del hormigón y después a intervalos pru- } \\
\text { denciales. }\end{array}$ & $\begin{array}{l}\text { Relación agua/cemento: En la } 1 .^{\mathrm{a}} \text { colocación, } \\
\text { después, aproximadamente, una vez al día. }\end{array}$ \\
\hline $\begin{array}{l}\text { Resistencia a la compresión: } 3 \text { cubos de cada } \\
500 \mathrm{~m}^{3} \text { y cada semana de hormigonado, ade- } \\
\text { más de cada piso en las construcciones ele- } \\
\text { vadas. } \\
\text { (Preferentemente para Bn } 150 \text { y Bn } 250 \text { ). }\end{array}$ & $\begin{array}{l}\text { Resistencia a la compresión : } 6 \text { cubos de cada } \\
500 \mathrm{~m}^{3} \text { y cada semana de hormigonado, ade- } \\
\text { más de cada piso en las construcciones ele- } \\
\text { vadas. La mitad de las determinaciones de } \\
\text { resistencia puede sustituirse por determina- } \\
\text { ciones de la relación agua/cemento. }\end{array}$ \\
\hline
\end{tabular}

Para hormigones con propiedades especiales se determinará el volumen de los ensayos en cada caso individual.

Según DIN 1045, apartado 8, para hormigón B II, hormigón preamasado y prefabricados de hormigón se exigirá un control de la calidad propio y ajeno, cuyos detalles se determinan en DIN 1084, hojas 1 a 3 (actualmente en proyecto).

*) Ver Betonstein Zeitung 36 (1970) n.* 8.

Para el hormigón son además imprescindibles las pruebas de idoneidad, calidad y endurecimiento. Las pruebas de idoneidad no son necesarias efectuarlas para el hormigón B I si su composición corresponde al apartado 3.1, párrafo 1 (tabla 3) y no contiene aditivo o adición alguna. En el ámbito de la prueba de calidad durante la fabricación continua se contrastarán los valores característicos de la composición del hormigón, así como las propiedades del hormigón fresco y endurecido. Las exigencias de la DIN 1045 sobre el tipo y volumen de estas comprobaciones se deducen tanto para hormigón B I como B II, de 
la tabla 14. Adquiriéndose hormigón preamasado, de la nota de entrega puede deducirse el contenido de cemento y la relación agua/cemento, y, en determinadas condiciones, a la prueba de calidad en obra pueden añadirse pruebas de resistencia del control propio de la estación de hormigonado. Las exigencias de resistencia en las pruebas de idoneidad $\mathrm{y}$ de calidad fueron tratadas ya en los apartados 2.3, 3.1 y 3.2. Hay que agregar todavía que las relaciones agua/cemento establecidas para la protección anticorrosiva de la armadura (apartado 5) y para propiedades especiales (apartado 6) no deben sobrepasarse en la prueba de calidad y que la relación agua/cemento fijada en la prueba de la idoneidad, para una resistencia determinada en la prueba de la calidad, puede sobrepasar como máximo el $10 \%$, aunque las tres relaciones particulares siguientes no deben sobrepasar el valor medio.

En DIN 1045, apartado 8, para hormigón B II, prefabricados de hormigón y hormigón preamasado, se exige un control de la calidad, compuesto de control propio y ajeno, cuyo volumen y particularidades se determinan teniendo en cuenta las disposiciones de la DIN 1045 en DIN 1084, hojas 1 a 3 (actualmente en proyecto).

La prueba del endurecimiento puede realizarse en probetas previstas al efecto con ensayos no destructivos según DIN 4240 (asimismo actualmente en revisión y probablemente sustituida por DIN 1048 hoja 2). En casos especiales está prevista una demostración de la resistencia del hormigón ya colocado en obra aportando un dictamen pericial (DIN 1045, apartado 7.4.5).

La norma DIN 1048 solamente contendrá datos sobre el modo en que han de efectuarse los ensayos. En la nueva versión se han revisado los métodos de ensayo existentes incluyendo como nuevos la determinación del aire ocluido, la medida de compacidad, la resistencia a la tracción y el módulo de elasticidad a compresión (este último ensayo en una hoja especial). Debido a que la relación agua/cemento es un factor influyente esencial, también se incluyen métodos para comprobar la composición del hormigón y la relación agua/cemento. Para el control durante la fabricación continua del hormigón hubo que hacer uso de métodos simplificados, como por ejemplo la determinación del contenido de agua (ensayo de Darr, ensayo de Thaulow).

\section{B I B L I O G R A F I A}

[1] Walz, K.: Weiterentwicklung der betontechnischen Bestiminungen. Der Bauingenieur 33 (1958) H. 1, S. 10/14 B] Bonzel, J.: Uber die neuere zement- und betontechnische Entwicklung. beton 17 (1967) H. 6, S. 221/224, und H. 7,
S. 263/267; ebenso Betontechnische Berichte 1967, Beton-Ver-

lag, Düsseldorf 1968, S. 63/83
[3] Walz, K., und J. Bonzel: Entwurf der Normen für Zuschläge, Beton und Stahlbeton (Betontechnologische Festlegungen). beton 18 (1968) H. 6, S. 217/220, und H. 7, S. 259/268; ebenso Betontechnische Berichte 1968, Beton-Verlag, Düssel-
dorf 1969 , S. 83/101

[4] Rüsch, H.: Der Einfluß der Streuung bei der Betonkon-

[4] trolle, Der Bauingenieur 37 (1962) H. 10, S. 373/377
[5] Rüsch, H.: Zur statistiscien Qualitatskontrolle des Be-

tons. Materialprüfung 6 (1964) $\mathrm{Nr}$. 11, S. $387 / 394$

[6] Bonzel, J., und W. Manns: Beurteilung der EctondruckH. 7, S. 303:307, und H. A, S. 355:360. ebenso Betontechnische H. 7, S. $303 \cdot 307$, und H. 8, S. 355:360; ebenso Betontechn
Berichte 1969 , Beton-Verlag, Düsseldorf 1970 , S. $85 / 114$
[7] Vorläufige Richtlinien für die Prüfung von Betonzusatzmitteln zur Erteilung von Prüfzeichen, Fassung Januar 1965 , mit Erläuterungen von W. Albrecht. Die. Bauwirtschaft 19
(1965) H. 6, S. 149/156

[3] Bonzel, J.: Beton mit hohem Frost- und Tausalzwiderstand. beton $15,(1965) \mathrm{H}, 11, \mathrm{~S} .468 / 474$, und $\mathrm{H}$. 12 , $\mathrm{S}$. 509/515; ebenso Betontechnische Berichte 1965, Beton-Verlag, Düs-
seldorf 1966, S. 185/216; ebenso Zement-Taschenbuch 1966.67, Bauverlag, Wiesbaden 1965, S. 305/343

[9] Bonzel, J., und F. W. Locher: Uber das Angriffsvermögen von Wässern, Böden und Gasen auf Beton. Anmerbeton 18 (1968) H. 10, S. 401 404, und H. 11, S. 443'445; ebenso Betontechnische Berichte 1968, Beton-Verlag, Düsseldorf

10] Merkblatt für die Herstellung geschlossener Betonoberflächen bei einer Wärmebehandlung mit Anmerkungen von
G. Wischers. beton 17 (1967) H. 3, S. 101/103, und H. 4, S. 139/142; ebenso Betontechnische Berichte 1967, Beton-Verlag, Düsseldorf 1968 , S. 35.52 